\title{
Honokiol ameliorates endothelial dysfunction through suppression of PTX3 expression, a key mediator of IKK/IкB/NF-KB, in atherosclerotic cell model
}

\author{
Ling Qiu ${ }^{1}$, Rong $\mathrm{Xu}^{1}$, Siyang Wang ${ }^{1}$, Shuijun $\mathrm{Li}^{2}$, Hongguang Sheng ${ }^{3}$, Jiaxi $\mathrm{Wu}^{2}$ and $\mathrm{Yi} \mathrm{Qu}^{1}$
}

Pentraxin 3 (PTX3) was identified as a marker of the inflammatory response and overexpressed in various tissues and cells related to cardiovascular disease. Honokiol, an active component isolated from the Chinese medicinal herb Magnolia officinalis, was shown to have a variety of pharmacological activities. In the present study, we aimed to investigate the effects of honokiol on palmitic acid (PA)-induced dysfunction of human umbilical vein endothelial cells (HUVECs) and to elucidate potential regulatory mechanisms in this atherosclerotic cell model. Our results showed that PA significantly accelerated the expression of

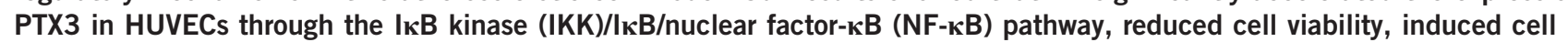
apoptosis and triggered the inflammatory response. Knockdown of PTX3 supported cell growth and prevented apoptosis by blocking PA-inducted nitric oxide (NO) overproduction. Honokiol significantly suppressed the overexpression of PTX3 in PA-inducted HUVECs by inhibiting IкB phosphorylation and the expression of two NF- $\mathrm{B}$ subunits (p50 and p65) in the $\mathrm{IKK} / \mathrm{l \kappa} / \mathrm{NF}-\kappa \mathrm{B}$ signaling pathway. Furthermore, honokiol reduced endothelial cell injury and apoptosis by regulating the expression of inducible NO synthase and endothelial NO synthase, as well as the generation of NO. Honokiol showed an antiinflammatory effect in PA-inducted HUVECs by significantly inhibiting the generation of interleukin-6 (IL-6), IL-8 and monocyte chemoattractant protein-1. In summary, honokiol repaired endothelial dysfunction by suppressing PTX3 overexpression in an atherosclerotic cell model. PTX3 may be a potential therapeutic target for atherosclerosis.

Experimental \& Molecular Medicine (2015) 47, e171; doi:10.1038/emm.2015.37; published online 3 July 2015

\section{INTRODUCTION}

Cardiovascular disease, a leading cause of mortality in developed countries, is mainly caused by atherosclerosis. Inflammatory response has a vital role in the pathogenesis of various cardiovascular diseases. ${ }^{1-6}$ Palmitic acid (PA) is the most common (saturated) fatty acid found in animals, plants and microorganisms. According to the World Health Organization, consumption of PA increases risk of developing cardiovascular diseases. It has been reported that PA induces oxidative stress and apoptosis in $\beta$ TC6 cells, ${ }^{7}$ impairs cell viability and increases apoptosis of neural stem cells by activating c-Jun N-terminal kinase. ${ }^{8}$ In addition, incubation with palmitic and linoleic acids at concentrations of $0.2 \mu \mathrm{M}$ or higher inhibited endothelial progenitor cell proliferation, significantly reduced migratory rate, reduced adhesion to fibronectin and impaired the ability of endothelial progenitor cells to form a tube structure. ${ }^{9}$ Consequently, PA is often used in vivo or in vitro, to model the effects of cardiovascular diseases. ${ }^{7-11}$
Pentraxins (PTXs) are an essential component of humoral immunity. Some research studies showed that PTX3, a prototypic member of the long PTX family, was connected to atherosclerosis. ${ }^{12,13}$ PTX3 was overexpressed in macrophages and endothelial cells in atherosclerotic tissue, and previous studies have also shown that PTX3 affected lipid accumulation in human macrophages. ${ }^{14,15}$ Clinical research has shown that the level of PTX3 was upregulated significantly in patients with unstable angina pectoris or acute coronary syndrome, and PTX3 was associated with coronary plaque vulnerability. ${ }^{16-18}$ Furthermore, in tissue samples of coronary artery disease, PTX3 deposits were found in the aorta and they were connected to the amount of inflammatory cell infiltrates in the tissue. ${ }^{19}$ At the same time, PTX3 has been linked to obesityassociated inflammation by serving as a cardioprotective modulator against cardiovascular disease. Higher levels of PTX3 were independently associated with increased mortality after ischemic stroke. It has been suggested that PTX3 is a

\footnotetext{
${ }^{1}$ Geriatrics Department, Shanghai Clinical Center, Chinese Academy of Sciences/Shanghai Xuhui Central Hospital, Shanghai, China; ${ }^{2}$ Shanghai Clinical Center, Chinese Academy of Sciences/Shanghai Xuhui Central Hospital, Shanghai, China and ${ }^{3}$ Endocrinology Department, Shanghai Clinical Center, Chinese Academy of Sciences/Shanghai Xuhui Central Hospital, Shanghai, China

Correspondence: Professor Y Qu, Geriatrics Department, Chinese Academy of Sciences/Shanghai Xuhui Central Hospital, 966 Huaihai Road (middle), Shanghai 200031, China. 
powerful prognostic biomarker in patients with ischemic stroke ${ }^{20,21}$ Overall, the level of PTX3 is confirmed to be closely associated with atherosclerosis. However, the correlation between endothelial cell injury and PTX3, and the regulatory mechanisms between upstream and downstream processes are still not fully understood.

Nuclear factor- $\mathrm{\kappa B}(\mathrm{NF}-\kappa \mathrm{B})$ has an important role in the intracellular regulation of immune response, inflammation and cell cycle. ${ }^{22-25} \mathrm{NF}-\mathrm{\kappa B}$ exists in the cytoplasm as an inactive $\mathrm{p} 50 /$ p65 protein heterodimer associated with an inhibitor protein.

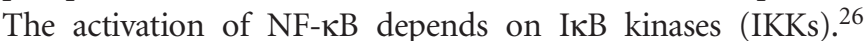
$\mathrm{NF}-\kappa \mathrm{B}$ and the associated downstream signaling pathway have been shown to mediate signaling in hyperglycemia or oxidative stress-triggered apoptosis of endothelial cells. ${ }^{27,28}$ It was reported that combining adipocyte-conditioned medium with oleic acid induces a synergistic NF- $\mathrm{KB}$ activation and leads to downregulation of NF- $\mathrm{KB}$ targets ang-1, SOD-1 and MMP-1 in human vascular smooth muscle cells. ${ }^{29}$ In addition, in $\mathrm{PTX}^{-1-}$ mice subjected to ischemia followed by reperfusion of the superior mesenteric artery, tissue injury was inhibited, and NF- $\mathrm{kB}$ translocation as well as cytokine production of CXCL1 and tumor necrosis factor- $\alpha$ (TNF- $\alpha$ ) were decreased. ${ }^{30}$ Therefore, the IKK/IкB/NF- $\mathrm{KB}$ signaling pathway might have an important role in the regulation of PTX3 expression and endothelial dysfunction.

Honokiol, an active component isolated from the Chinese medicinal herb M. officinalis, has been shown to possess a variety of pharmacological activities. ${ }^{31-37}$ Recent studies have shown that honokiol suppressed TNF- $\alpha$-induced migration and matrix metalloproteinase expression by blocking NF- $\mathrm{\kappa B}$ activation via the ERK signaling pathway in rat aortic smooth muscle cells. ${ }^{37}$ Honokiol caused the p21WAF1-mediated G1 phase arrest of the cell cycle by inducing p 38 mitogen-activated protein kinase in vascular smooth muscle cells. ${ }^{38}$ Honokiol has also alleviated hyperglycemia, hyperlipidemia, hepatic oxidative damage and insulin resistance in diabetic rats by inhibiting hepatic CYP2E1 activity. ${ }^{39}$ In addition, honokiol upregulated prostacyclin synthase protein expression and inhibited endothelial cell apoptosis. ${ }^{40}$ Although honokiol has a role in anti-inflammatory, antioxidant, anti-angiogenic and antitumor activity in certain cell types, its effects on PA-inducted human umbilical vein endothelial cells (HUVECs) are unclear.

In this study, we incubated HUVECs with PA to establish an endothelial cell injury model. We aimed to investigate the function of honokiol and to elucidate regulatory mechanisms in this atherosclerotic cell model. The phosphorylation level of I KB and the expression of two NF-KB subunits (p50 and p65) in the $\mathrm{IKK} / \mathrm{I} \kappa \mathrm{B} / \mathrm{NF}-\mathrm{\kappa B}$ signaling pathway were determined with western blotting. The level of inflammatory cytokines (interleukin-6 (IL-6), IL-8 and monocyte chemoattractant protein-1 (MCP-1)) and vascular endothelium active molecules (nitric oxide (NO), induced NO synthase (iNOS) and endothelial NO synthase (eNOS)) were evaluated using western blotting and enzyme-linked immunosorbent assay measurement.

\section{MATERIALS AND METHODS}

\section{Cell culture}

HUVECs were obtained from the Cell Resource Center at the Shanghai Institutes for Biological Sciences (SIBS, Shanghai, China) and cultured in RPMI 1640 medium supplemented with $10 \%$ fetal bovine serum (Invitrogen, Carlsbad, CA, USA). The cells were washed and incubated in endotoxin-free RPMI 1640 (10\% fetal bovine serum) at $37^{\circ} \mathrm{C}$ in the presence of $5 \% \mathrm{CO}_{2}$.

\section{Cell viability assay and cell apoptosis assay}

Cells were digested and reseeded in 96-well plates for a cell viability assay, by using the Cell Counting Kit (CCK-8, Beyotime, Shanghai, China), according to the manufacturer's protocol.

Apoptosis was measured using an annexin-V-Fluos and propidium iodide Apoptosis Detection Kit (Beyotime) and a flow cytometer (Becton Dickinson Diagnostic Systems, Sparks, MD, USA), according to the manufacturer's guidelines.

Quantitative reverse-transcriptase PCR and western blotting Total RNA was extracted from cells using TRIzol (Invitrogen) and reverse transcribed into cDNA using the SuperScriptIII reversetranscriptase kit (Invitrogen), according to the manufacturer's instructions. Quantitative reverse-transcriptase PCR for PTX3 mRNA level was performed using the SYBR Green Master (Invitrogen) on a Stratagene MX3005P system (Agilent Technologies, Santa Clara, CA, USA). $\beta$-Actin served as an internal standard. Relative gene expression was calculated using the $2^{-\Delta \Delta \mathrm{Ct}}$ method. ${ }^{41}$

Protein extracted from cells were separated by $10 \%$ SDSpolyacrylamide gel electrophoresis and transferred to polyvinylidene difluoride membranes (Millipore, Schwalbach, Hessen, Germany). Membranes were blocked and then incubated with primary antibodies specific for PTX3, IкB- $\alpha, \mathrm{p}-\mathrm{I \kappa B}-\alpha, \mathrm{p} 50$, p65, iNOS and eNOS. $\beta$-Actin was used as a protein loading control. The membranes were incubated with the appropriate HRP (horseradish peroxidase)-conjugated antibody and visualized with chemiluminescence (Thermo Scientific, Rockford, IL, USA).

\section{RNA interference}

Three different small interfering RNA were synthesized by Genepharma (Shanghai, China) and transfected into HUVECs, to suppress the function of PTX3 using Lipofectamine 2000 (Invitrogen).

\section{Enzyme-linked immunosorbent assay measurement}

HUVECs were exposed to PA (0.5 mm, Sigma, St Louis, MO, USA), PA+honokiol $(10 \mu \mathrm{m}$, Shanghai yuanye Bio-Technology, Shanghai, China) or PA+TPCA-1 (30 $\mu$, Selleck, Houston, TX, USA) for $48 \mathrm{~h}$, and the culture medium was collected. The amount of PTX3 (Dakewe Biotech, Shanghai, China), MCP-1 (Abcam, Cambridge, UK), NO (Dakewe Biotech), IL-6 (Dakewe Biotech) and IL-8 (Dakewe Biotech) were quantified using a human enzyme-linked immunosorbent assay kit, according to the manufacturer's instructions. The absorbance values were read at $450 \mathrm{~nm}$ in a microplate reader (BioTek, Winooski, VT, USA).

\section{Immunohistochemical analysis}

HUVECs were incubated in 96-well plates with PA $(0.5 \mathrm{~mm})$ or honokiol $(10 \mu \mathrm{M})$ for $48 \mathrm{~h}$. The cells were washed with phosphatebuffered saline, fixed with $4 \%$ paraformaldehyde and permeabilized in $0.1 \%$ Triton X-100, then blocked with $1 / 100$ diluted goat serum and 
incubated overnight at $4{ }^{\circ} \mathrm{C}$ with an anti-PTX3 primary antibody (Santa Cruz Biotechnology, Dallas, TX, USA). After two washes with phosphate-buffered saline, the cells were incubated for $1 \mathrm{~h}$ at $37^{\circ} \mathrm{C}$ with a secondary antibody (Santa Cruz Biotechnology). Cells were washed again and stained with DAPI (2-(4-amidinophenyl)- $1 \mathrm{H}$ -indole-6-carboxamidine). The samples were visualized with fluorescence microscopy (Carl Zeiss, Jena, Germany).

\section{Statistical analysis}

Statistical analysis was performed using SPSS software version 16.0 (SPSS Inc., Chicago, IL, USA). All experiments were performed at least three times and the data are shown as the mean \pm s.d. A two-tailed $t$ test was used for comparing two independent groups. One-way analysis of variance was used to compare multiple groups. $P<0.05$ was considered statistically significant.

\section{RESULTS}

PA induced HUVEC apoptosis and PTX3 overexpression.

To investigate PA-induced injury of HUVECs, we incubated the HUVECs with different concentrations of PA. The viability of HUVECs incubated with different concentrations of PA for 24 or $48 \mathrm{~h}$ was determined using CCK- 8 assays. The results showed that PA treatment induced cell death in a concentration-dependent and time-dependent manner (Figure 1a). To investigate whether PA induced cell death through an apoptotic mechanism, annexin V/propidium iodide double staining was used to detect early apoptosis. As shown in Figure $1 c$, the early apoptotic rate significantly increased in
HUVECs after $48 \mathrm{~h}$ of PA exposure at doses of 0.5 and $1 \mathrm{~mm}$ $(P<0.05)$. Finally, we investigated the level of PTX3 in HUVECs with PA treatment. The quantitative reversetranscriptase PCR and western blot analyses showed that the expression level of mRNA and protein for PTX3 significantly increased in the PA treatment group compared with the control (Figure 2a). In addition, immunochemistry exhibited an enhanced PTX3 signal in HUVECs after PA treatment (Figure 2b).

At the same time, we incubated HUVECs with different concentrations of honokiol for 24 or $48 \mathrm{~h}$, to detect the effect of honokiol on HUVEC viability. The results showed that HUVECs had almost the same viability when they were exposed to honokiol concentrations of $0-30 \mu \mathrm{M}$ for $24 \mathrm{~h}$. Concentrations higher than $40 \mu \mathrm{M}$ markedly altered cell viability (Figure $1 \mathrm{~b}, P<0.05$ ). In the 48 -h exposure group, at concentrations $>10 \mu \mathrm{M}$, honokiol significantly inhibited cell viability (Figure $1 \mathrm{~b}, P<0.05$ ). Consequently, we selected a concentration of $10 \mu \mathrm{M}$ for the next experiment. As shown in Figures $1 \mathrm{c}$ and 2, compared with PA treatment $(0.5 \mathrm{~mm})$, honokiol $(10 \mu \mathrm{M})$ significantly inhibited cell apoptosis and PTX3 expression $(P<0.05)$.

Overall, we successfully established a PA-induced HUVEC injury model for atherosclerosis research and determined the expression of PTX3 was upregulated in HUVECs by PA. a

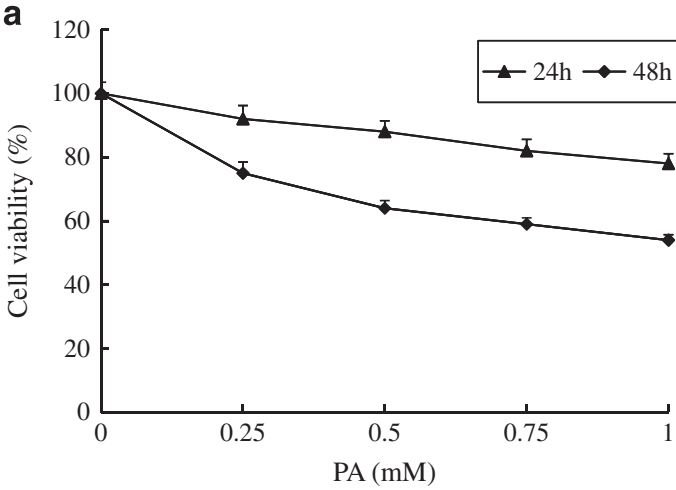

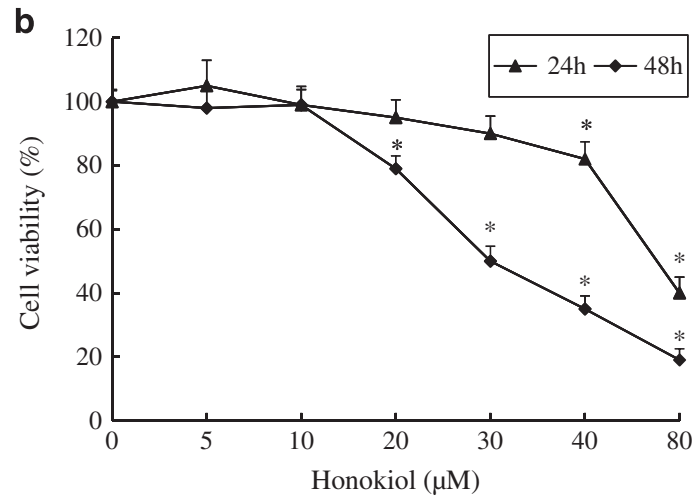
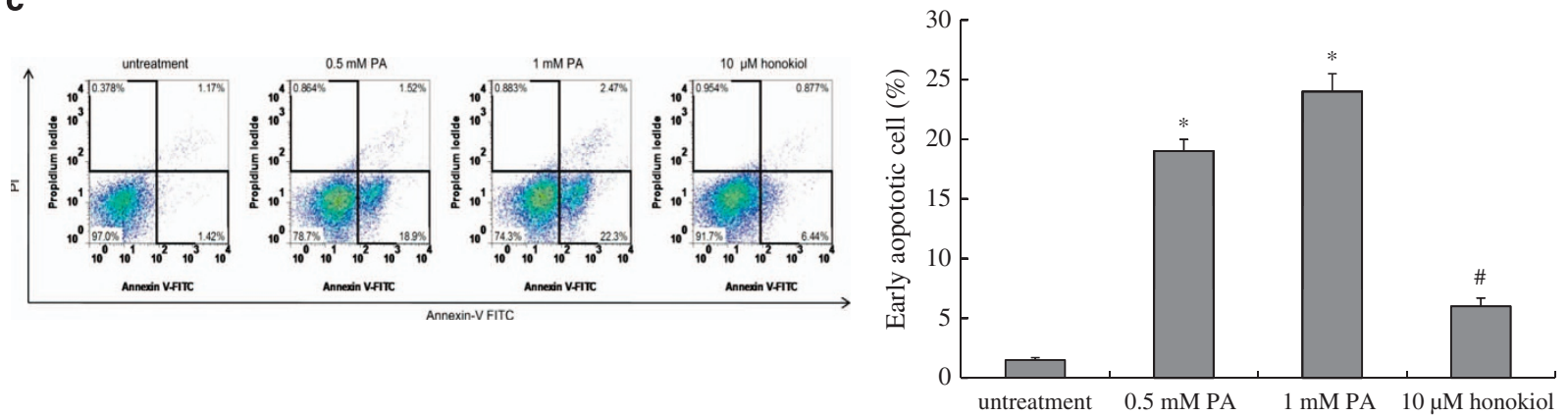

Figure 1 PA-induced HUVECs injury. (a) Cell viability of HUVECs after treatment with various concentrations of PA for 24 or $48 \mathrm{~h}$. (b) Cell viability of HUVECs after treatment with various concentrations of honokiol for 24 or $48 \mathrm{~h}$. (c) Apoptosis of HUVECs after treatment with vehicle, $0.5 \mathrm{~mm}$ PA, $1 \mathrm{~mm}$ PA or $10 \mu \mathrm{m}$ honokiol for $48 \mathrm{~h}$. Data are mean \pm s.d., $n=3$ in each group. ${ }^{*} P<0.05$, versus untreated group. $\# P<0.05$, versus $0.5 \mathrm{~mm}$ PA treatment group. 


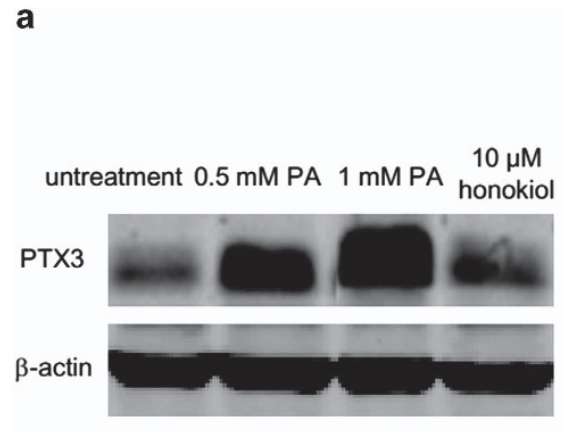

b

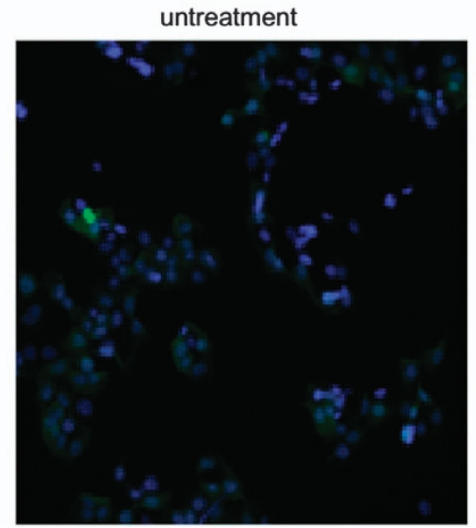

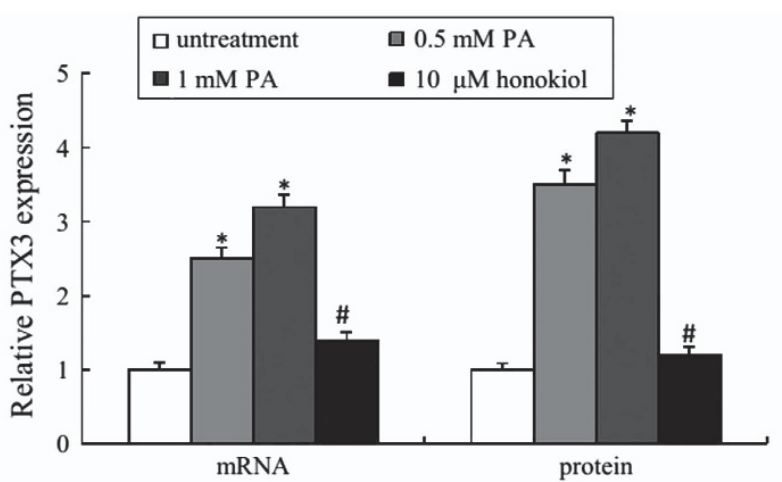

$0.5 \mathrm{mM} P A$



$10 \mu \mathrm{M}$ honokiol

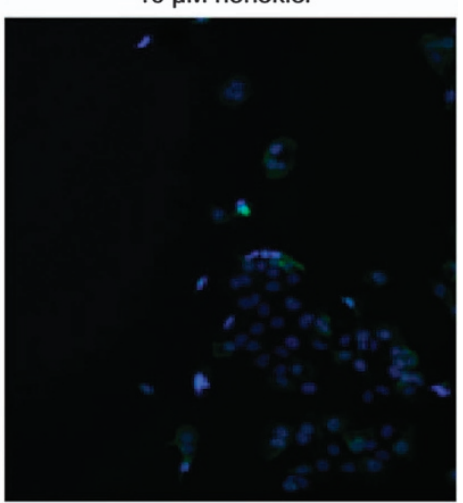

Figure 2 PA-induced PTX3 overexpression. (a) Protein and mRNA expression of PTX3 in HUVECs after treatment with vehicle, 0.5 mm PA, $1 \mathrm{~mm}$ PA or $10 \mu \mathrm{m}$ honokiol for $48 \mathrm{~h}$. (b) Immunohistochemical staining of PTX3 in HUVECs after treatment with vehicle, $0.5 \mathrm{~mm}$ PA or $10 \mu \mathrm{m}$ honokiol for $48 \mathrm{~h}$. Data are mean \pm s.d., $n=3$ in each group. ${ }^{*} P<0.05$, versus untreated group. $\# P<0.05$, versus $0.5 \mathrm{~mm}$ PA treatment group.

\section{Honokiol suppressed PA-induced overexpression of PTX3 and inflammatory response in HUVECs}

PA caused dysfunction in HUVECs and led to overexpression of PTX3. However, the regulatory mechanism of PTX3 expression in PA-inducted HUVECs remains unclear. The NF-KB pathway has been shown to have an important role in the inflammatory response, especially in tumorigenesis caused by chronic inflammation. ${ }^{42,43}$ To understand the possible mechanism behind PTX3 overexpression in a PAinduced atherosclerosis model, we incubated HUVECs with PA $(0.5 \mathrm{mM})$, PA plus an inhibitor of IKK-2 (TPCA-1, $30 \mu \mathrm{M})$ or PA plus honokiol $(10 \mu \mathrm{M})$ for $48 \mathrm{~h}$. From enzyme-linked immunosorbent assay analysis, quantitative reversetranscriptase PCR and western blotting, we observed an obvious increase of PTX3 in HUVECs after PA treatment $(P<0.05$, Figures $3 \mathrm{a}, \mathrm{b}$ and $\mathrm{c})$. In addition, TPCA- 1 , an inhibitor of IKK-2, significantly suppressed the upregulation of PTX3 induced by PA $(P<0.01)$. Similarly, honokiol significantly inhibited the ectopic expression of PTX3 in HUVECs $(P<0.01)$.

Next, we investigated the activation of NF- $\mathrm{\kappa B}$ by performing western blotting. The phosphorylation level of IкB- $\alpha$ is shown in Figure 3d. Compared with the untreated group, the phosphorylation levels of IкB- $\alpha$ increased dramatically under PA treatment $(P<0.05)$. Similarly, the expression levels of two NF- $\mathrm{kB}$ subunits, p50 and p65, were significantly increased in the nucleus after exposure to PA $(P<0.05$, Figure 3e). Conversely, honokiol significantly inhibited the phosphorylation of I $\kappa \mathrm{B}$ compared with PA treatment. The levels of p50 and p65 also decreased after honokiol treatment $(P<0.05)$.

The results indicate that PA-inducted PTX3 overexpression occurred through the IKK/IKB/NF- $\mathrm{kB}$ signaling pathway. After PA treatment, the phosphorylation level of I $\kappa B$ increased, NF- $\kappa B$ was activated and transferred from cytoplasm into the nucleus and it combined with the corresponding DNA segments to regulate PTX3 transcription and expression. In this process, honokiol might operate as a kinase inhibitor that effectively suppresses PTX3 overexpression.

In addition, the inflammatory response induced by PA was evaluated by measuring the level of two indicators (IL-6 and IL-8) and MCP-1. The results showed that PA significantly increased the levels of IL-6, IL-8 and MCP-1 in HUVECs $(P<0.05)$, which suggests that PA could cause an inflammatory response in HUVECs (Figure 3f). However, honokiol treatment led to a significant reduction of IL-6, IL-8 and MCP-1 $(P<0.05)$, which indicates that honokiol could function as an anti-inflammatory agent in PA-induced HUVECs.

\section{Honokiol inhibits PA-induced HUVECs injury and apoptosis}

In HUVECs, PA upregulated PTX3 expression, suppressed cell growth and induced cell apoptosis. To investigate the role of 
a

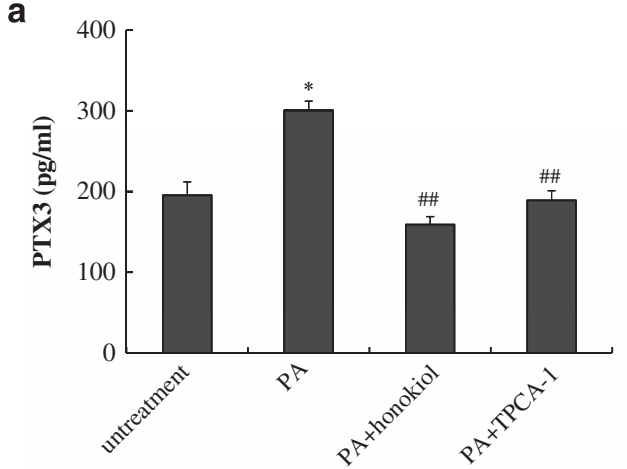

b
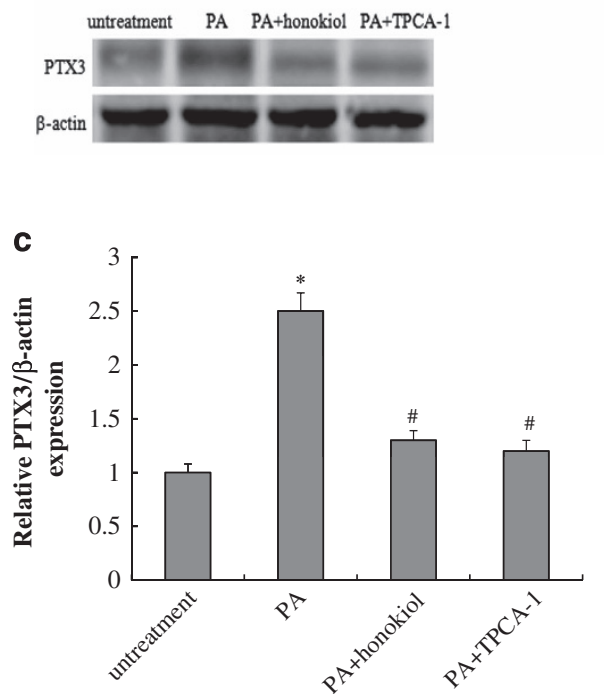

d
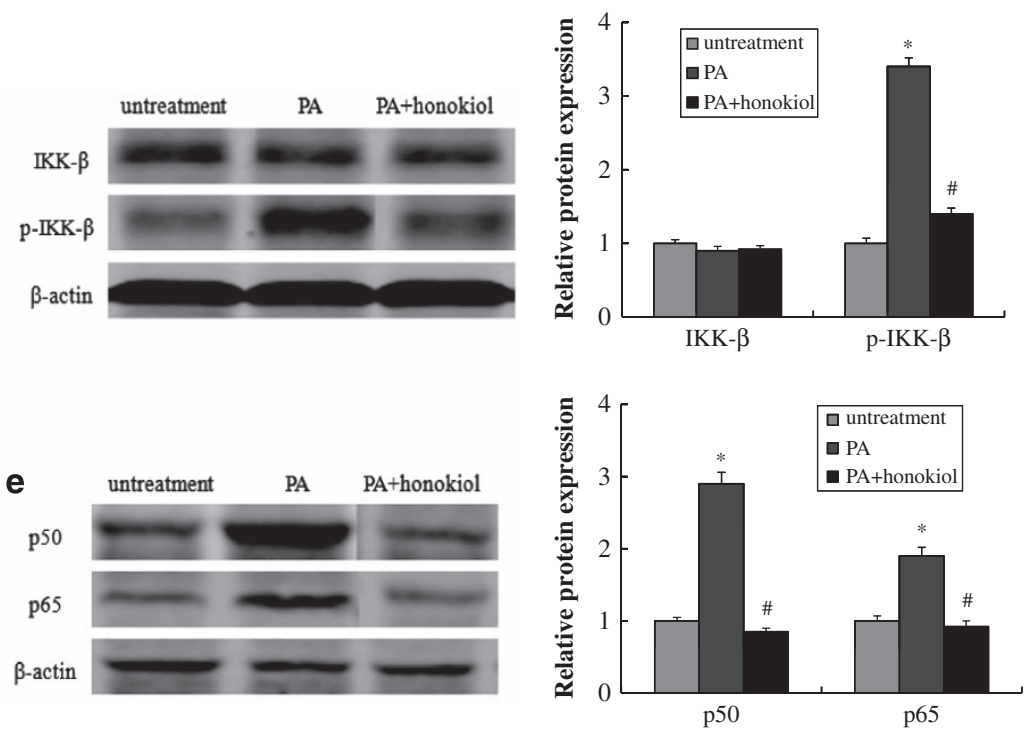

\section{f}

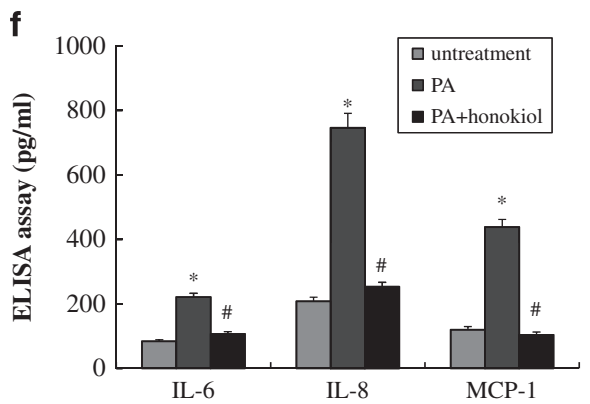

Figure 3 Honokiol regulated the expression of PTX3 and inflammatory response in PA-induced HUVECs model. PTX-3 levels from the enzyme-linked immunosorbent assay (ELISA) (a), western blotting (b) and reverse-transcriptase PCR (RT-PCR) (c) in HUVECs after treatment with vehicle, $0.5 \mathrm{~mm}$ PA, PA plus $10 \mu \mathrm{m}$ honokiol or PA plus an inhibitor of IKK-2 (TPCA-1, $30 \mu \mathrm{m}$ ) for $48 \mathrm{~h}$. (d) Protein expression of IkB and p-IkB in HUVECs after treatment with vehicle, $0.5 \mathrm{~mm}$ PA or PA plus $10 \mu \mathrm{m}$ honokiol for $48 \mathrm{~h}$. (e) Protein expression of p50 and p65 in HUVECs after treatment with vehicle, $0.5 \mathrm{~mm}$ PA or PA plus $10 \mu \mathrm{m}$ honokiol for $48 \mathrm{~h}$. (f) IL-6, IL-8 and MCP-1 levels measured by the ELISA assay in HUVECs after treatment with vehicle, $0.5 \mathrm{~mm}$ PA or PA plus $10 \mu \mathrm{m}$ honokiol for $48 \mathrm{~h}$. Data are mean $\pm \mathrm{s}$. d., $n=3$ in each group. ${ }^{*} P<0.05$, versus untreated group. ${ }^{\#} P<0.05$ and ${ }^{\# \#} P<0.01$, versus 0.5 mm PA treatment group.

PTX3 in PA-induced HUVECs apoptosis, we blocked PTX3 with small interfering RNA. After treatment with PA $(0.5 \mathrm{~mm})$, eNOS expression significantly decreased and iNOS expression increased compared with the untreated group $(P<0.05$, Figure 4a). Accordingly, NO production also increased $(P<0.05$, Figure $4 \mathrm{~b})$. Knockdown of PTX3 led to a significant rise of eNOS and a reduction in iNOS, as well as a significant reduction in NO secretion $(P<0.05$, Figures $4 \mathrm{a}$ and $\mathrm{b})$. The results of the cell viability and apoptosis analyses showed that knockdown of PTX3 repaired cell growth and blocked cell apoptosis induced by PA (Figures $4 \mathrm{c}$ and d). Collectively, these results suggest that PTX3 was one of the targets of PA-induced apoptosis. A potential regulation mechanism was that PTX3 inhibited eNOS expression and activated iNOS expression, thereby promoting the overproduction of NO in HVECs and eventually leading to apoptosis.
Furthermore, we found that honokiol could inhibit PAinducted vascular endothelial cell injury and apoptosis through the NO pathway. Compared with PA treatment, honokiol $(10 \mu \mathrm{M})$ significantly increased eNOS protein levels, decreased iNOS protein levels, inhibited NO production and prevented cell apoptosis $(P<0.05$, Figure 4$)$.

\section{DISCUSSION}

In the present study, we found that PTX3 is a target of PAinduced endothelial cell injury. Honokiol had an antiinflammatory effect and repaired endothelial dysfunction by suppressing PTX3 expression via the IKK/ІкB/NF-кB pathway in an atherosclerotic cell model. Our results strongly suggest that honokiol may be used clinically in the near future to treat patients with endothelial dysfunction and PTX3 may also be a potential therapeutic target for treating atherosclerosis. 
a

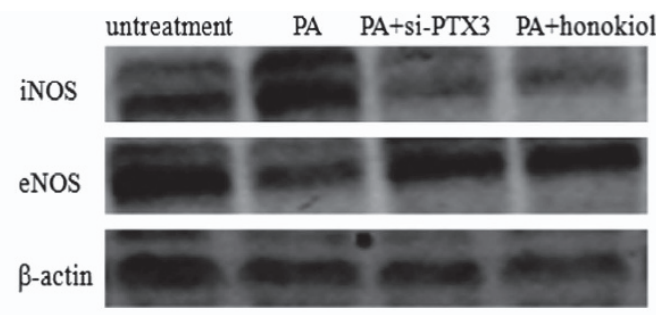

b

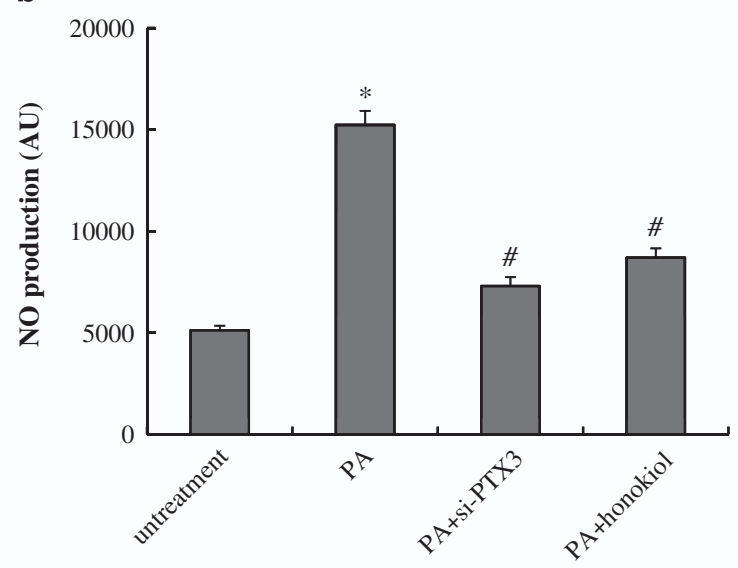

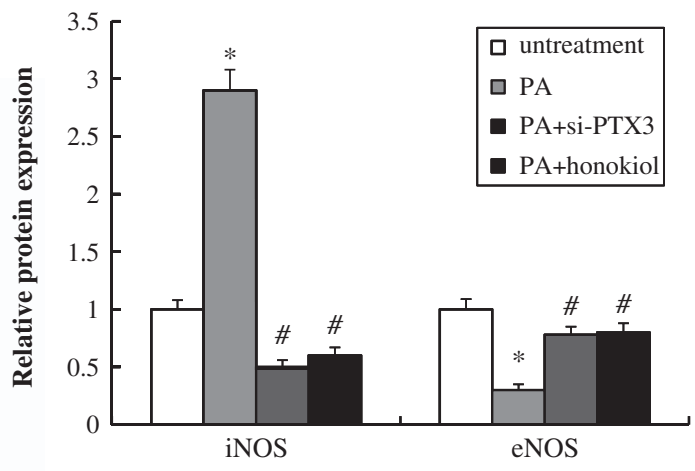

C
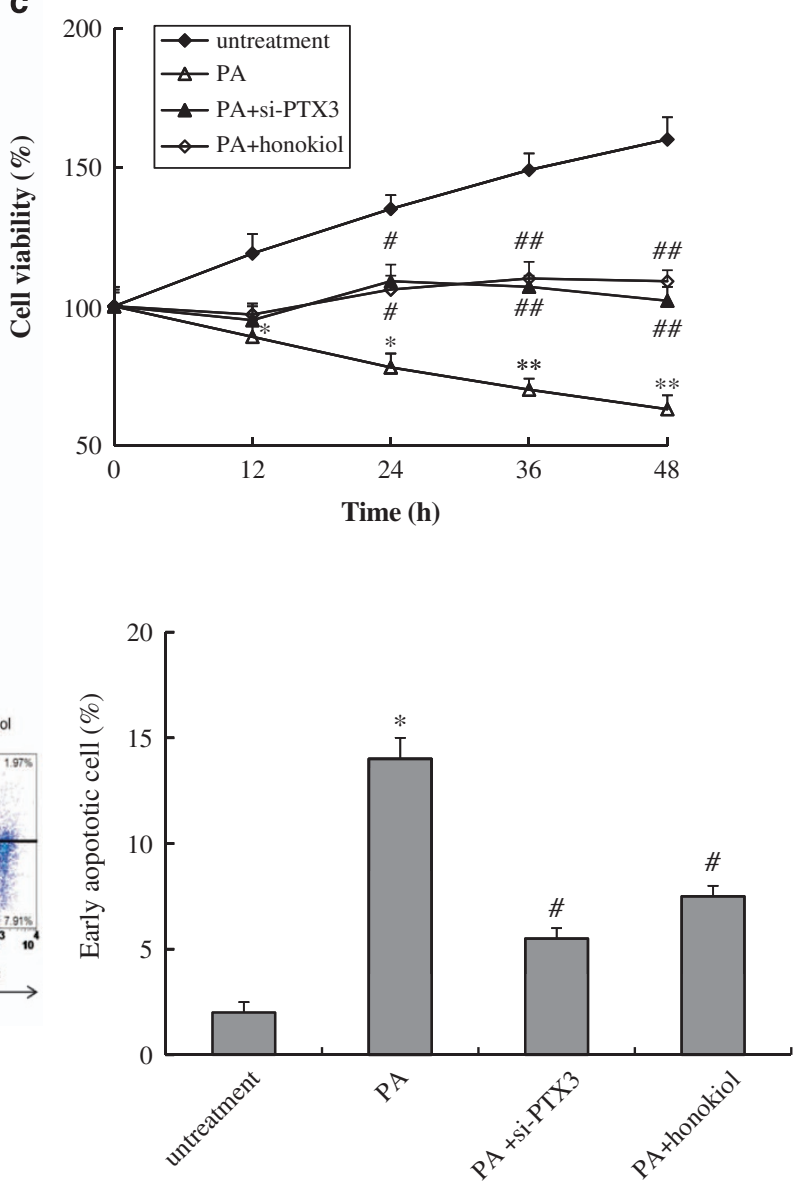

Figure 4 Effect of PTX3 knockdown on PA-induced HUVECs model. (a) Protein expression of iNOS and eNOS, and (b) NO concentration in HUVECs after treatment with vehicle, $0.5 \mathrm{~mm}$ PA, PA plus $10 \mu \mathrm{m}$ honokiol or si-PTX3 plus $0.5 \mathrm{~mm}$ PA for $48 \mathrm{~h}$. (c) The cell viability and (d) apoptosis of HUVECs after treatment with vehicle, $0.5 \mathrm{~mm}$ PA, PA plus $10 \mu \mathrm{m}$ honokiol or si-PTX3 plus $0.5 \mathrm{~mm}$ PA for $48 \mathrm{~h}$. Data are mean \pm s.d., $n=3$ in each group. ${ }^{*} P<0.05$, ${ }^{*} P<0.01$, versus untreated group. ${ }^{\#} P<0.05$, \#\# $P<0.01$, versus $0.5 \mathrm{~mm} P A$ treatment group.

In HUVECs, PA treatment induced cell apoptosis and PTX3 overexpression. PTX3 was confirmed to be a key mediator of

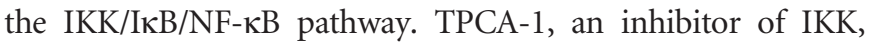
significantly suppressed PA-induced overexpression of PTX3 in HUVECs at the protein level and the mRNA level. The results of our RNA interference experiment showed that knockdown of PTX3 repaired cell growth and prevented cell apoptosis by regulating the levels of iNOS/eNOS and the generation of NO, whereas the opposite results occurred when PTX3 was overexpressed. The findings suggest that PTX3 might have an important role in PA-induced HUVECs apoptosis. These experiments provided evidence that PTX3 is associated with atherosclerosis and might be a potential therapeutic target for atherosclerosis.

Honokiol is a small-molecule polyphenol isolated from the Chinese medicinal herb M. officinalis. In the last few years, honokiol has been found to have anti-angiogenic, antiinflammatory and anti-tumor properties in preclinical 
models. ${ }^{31-36}$ It was reported that honokiol reduced secretion of IL-8 and TNF- $\alpha$ induced by Propionibacterium acnes in THP-1 cells. ${ }^{33}$ Honokiol inhibited TNF- $\alpha$-induced RASMC proliferation and migration in a dose-dependent manner and blocked TNF- $\alpha$-induced protein expression of MMP- 2 and MMP-9, NF- $\kappa B$ activation and ERK1/2 phosphorylation. ${ }^{37}$ In the present work, we found that honokiol significantly reduced inflammatory cytokines, IL-6 and IL-8, as well as MCP-1, and it demonstrated an anti-inflammatory effect on PA-inducted HUVECs.

Other studies have suggested that honokiol suppresses increases in NADPH oxidase activity, Rac-1 phosphorylation, p22phox protein expression and the production of reactive oxygen species, by inhibiting upregulation of high glucose (HG)-induced NF-kB-regulated COX-2, apoptosis and cell death in HUVECs. ${ }^{44}$ Zhang et al. ${ }^{45}$ found that honokiol significantly reduced the level of the p65 subunit of NF- $\mathrm{\kappa B}$ in the nucleus of primary-culture microglia. In our study, honokiol had a kinase inhibitor role by significantly suppressing the overexpression of PTX3 in PA-induced HUVECs through inhibiting $\mathrm{I} \kappa \mathrm{B}$ phosphorylation and the protein expression of two NF- $\mathrm{KB}$ subunits (p50 and p65) in the $\mathrm{IKK} / \mathrm{I \kappa B} / \mathrm{NF}-\mathrm{\kappa B}$ signaling pathway. Ahn et al. ${ }^{46}$ found that honokiol affects NF- $\mathrm{KB}$ signaling through an indirect effect on NF-kB/DNA binding. Honokiol inhibited TNF-induced NF- $\mathrm{kB}$

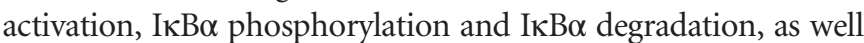
as inhibiting NF- $\mathrm{KB}$-dependent reporter gene expression induced by TNFR1, TRADD, TRAF, NIK and IKK $\beta$. Finally, honokiol decreased levels of NF- $\mathrm{KB}$ target genes.

Furthermore, our study showed that honokiol could effectively inhibit PA-induced HUVECs apoptosis through the NO pathway. Honokiol significantly reduced iNOS levels and activated eNOS expression to block the overproduction of NO. It was reported that $0.1-10 \mu \mathrm{m}$ concentrations of honokiol reduced NO levels in glial cells. ${ }^{45} \mathrm{Ou}$ et al. ${ }^{47}$ also showed that honokiol affected eNOS protein expression, attenuated cell apoptosis and affected reactive oxygen species generation in oxLDL-induced HUVECs. Recently, it was reported that rutin improved endothelial function by augmenting NO production in HUVECs. ${ }^{48} \mathrm{NO}$ is an important vascular endothelium active molecule and a moderate concentration of NO can protect endothelial functions. In the $\mathrm{NO}$ pathway, guanylate cyclase and are both the downstream targets of NO. Guanylate cyclase can improve the cyclic GMP level and NF- $\mathrm{KB}$ is an important transcription factor for iNOS expression.

In summary, in a PA-inducted HUVEC injury model, honokiol suppressed the overexpression of PTX3 by inhibiting I $\kappa \mathrm{B}$ phosphorylation and NF- $\mathrm{KB}$ activation in the $\mathrm{IKK} / \mathrm{I} \mathrm{\kappa B} / \mathrm{NF}-\kappa \mathrm{B}$ signaling pathway, thereby impacting the expression of iNOS/eNOS and the production of NO, as well as repairing endothelial dysfunction. However, the endocrine system is a very complex system and the pathogenesis of each disease generally involves multiple signaling pathways. The present study had partial limitations, because we only investigated the regulatory mechanism of honokiol in the classic IKK/IкB/NF- $\mathrm{KB}$ signaling pathway and only discussed the role of HUVECs. If necessary, we can conduct more comprehensive work in the future to systematically evaluate the function of honokiol in different types of cells and multiple pathway. Honokiol suppressed the PA-induced overexpression of PTX3 by inhibiting I $\mathrm{B}$ phosphorylation and NF- $\kappa \mathrm{B}$ activation in the $\mathrm{IKK} / \mathrm{I \kappa B} / \mathrm{NF}-\kappa \mathrm{B}$ signaling pathway, regulating the expression of iNOS/eNOS and the production of NO, as well as repairing endothelial dysfunction in the PA-induced HUVECs model. PTX3 may be a potential therapeutic target for treating atherosclerosis.

\section{CONFLICT OF INTEREST}

The authors declare no conflict of interest

\section{ACKNOWLEDGEMENTS}

This work was supported by Shanghai Municipal Health Bureau Program 20124276 and Shanghai XuHui District Health Bureau Program SHXH201203.

1 Klingenberg R, Hansson GK. Treating inflammation in atherosclerotic cardiovascular disease: emerging therapies. Eur Heart J 2009; 30: 2838-2844.

2 Libby $\mathrm{P}$, Okamoto $\mathrm{Y}$, Rocha VZ, Folco E. Inflammation in atherosclerosis: transition from theory to practice. Circ J 2010; 74: 213-220.

3 Zwaka TP, Hombach V, Torzewski J. C-reactive protein-mediated low density lipoprotein uptake by macrophages: implications for atherosclerosis. Circulation 2001; 103: 1194-1197.

4 Stoner L, Lucero AA, Palmer BR, Jones LM, Young JM, Faulkner J. Inflammatory biomarkers for predicting cardiovascular disease. Clin Biochem 2013; 46: 1353-1371.

5 Hagihara H, Nomoto A, Mutoh S, Yamaguchi I, Ono T. Role of inflammatory responses in initiation of atherosclerosis: effects of anti-inflammatory drugs on cuff-induced leukocyte accumulation and intimal thickening of rabbit carotid artery. Atherosclerosis 1991; 91: 107-116.

6 Black $\mathrm{PH}$. The inflammatory response is an integral part of the stress response: Implications for atherosclerosis, insulin resistance, type II diabetes and metabolic syndrome X. Brain Behav Immun 2003; 17: 350-364.

7 Shen X, Yang L, Yan S, Wei W, Liang L, Zheng $\mathrm{H}$ et al. The effect of FFAR1 on pioglitazone-mediated attenuation of palmitic acid-induced oxidative stress and apoptosis in $\beta$ TC6 cells. Metabolism 2014; 63: 335-351.

8 Yuan Q, Zhao S, Wang F, Zhang H, Chen ZJ, Wang J et al. Palmitic acid increases apoptosis of neural stem cells via activating c-Jun N-terminal kinase. Stem Cell Res 2013; 10: 257-266.

9 Guo WX, Yang QD, Liu YH, Xie XY, Wang-Miao, Niu RC. Palmitic and linoleic acids impair endothelial progenitor cells by inhibition of Akt/eNOS pathway. Arch Med Res 2008; 9: 434-442.

10 Wong KL, Wu YR, Cheng KS, Chan P, Cheung CW, Lu DY et al. Palmitic acid-induced lipotoxicity and protection by $(+)$-catechin in rat cortical astrocytes. Pharmacol Rep 2014; 66: 1106-1113.

11 Oh JM, Choi JM, Lee JY, Oh SJ, Kim HC, Kim BH et al. Effects of palmitic acid on TNF- $\alpha$-induced cytotoxicity in SK-Hep-1 cells. Toxicol In Vitro 2012; 26: 783-790.

12 Norata GD, Garlanda C, Catapano AL. The long pentraxin PTX3: a modulator of the immunoinflammatory response in atherosclerosis and cardiovascular diseases. Trends Cardiovasc Med 2010; 20: 35-40.

13 Alberti L, Gilardini L, Zulian A, Micheletto G, Peri G, Doni A et al. Expression of long pentraxin PTX3 in human adipose tissue and its relation with cardiovascular risk factors. Atherosclerosis 2009; 202: 455-460.

14 Liu W, Jiang J, Yan D, Li D, Li W, Ma Y et al. Pentraxin 3 promotes oxLDL uptake and inhibits cholesterol efflux from macrophage-derived foam cells. Exp Mol Pathol 2014; 96: 292-299.

15 Rolph MS, Zimmer S, Bottazzi B, Garlanda C, Mantovani A, Hansson GK. Production of the long pentraxin PTX3 in advanced atherosclerotic plaques. Arterioscler Thromb Vasc Biol 2002; 22: e10-e14. 
16 Inoue K, Sugiyama A, Reid PC, Ito Y, Miyauchi K, Mukai S et al Establishment of a high sensitivity plasma assay for human pentraxin3 as a marker for unstable angina pectoris. Arterioscler Thromb Vasc Biol 2007; 27: 161-167.

17 Eggers KM, Armstrong PW, Califf RM, Johnston N, Simoons ML, Venge P et al. Clinical and prognostic implications of circulating pentraxin 3 levels in non ST-elevation acute coronary syndrome. Clin Biochem 2013; 46: 1655-1659.

18 Soeki T, Niki T, Kusunose K, Bando S, Hirata Y, Tomita N et al. Elevated concentrations of pentraxin 3 are associated with coronary plaque vulnerability. J Cardiol 2011; 58: 151-157.

19 Hollan I, Nebuloni M, Bottazzi B, Mikkelsen K, Førre ØT, Almdahl SM et al. Pentraxin 3, a novel cardiovascular biomarker, is expressed in aortic specimens of patients with coronary artery disease with and without rheumatoid arthritis. Cardiovasc Pathol 2013; 22: 324-331.

20 Ryu WS, Kim CK, Kim BJ, Kim C, Lee SH, Yoon BW. Pentraxin 3: a novel and independent prognostic marker in ischemic stroke. Atherosclerosis 2012; 220(2): 581-586.

21 Slusher AL, Mock JT, Whitehurst M, Maharaj A, Huang CJ. The impact of obesity on pentraxin 3 and inflammatory milieu to acute aerobic exercise. Metabolism 2015; 64: 323-329.

22 Zhang M, Xiao Z, Sun L. Overexpression of NF-kB inhibitor alpha in Cynoglossus semilaevis impairs pathogen-induced immune response. Dev Comp Immunol 2012; 36: 253-257.

23 Lin T, Tamaki Y, Pajarinen J, Waters HA, Woo DK, Yao Z et al. Chronic inflammation in biomaterial-induced periprosthetic osteolysis: NF- $\mathrm{KB}$ as a therapeutic target. Acta Biomater 2014; 10: 1-10.

24 Ishak DHA, Ooi KK, Ang KP, Akim AM, Cheah YK, Nordin N et al. A bismuth diethyldithiocarbamate compound promotes apoptosis in HepG2 carcinoma, cell cycle arrest and inhibits cell invasion through modulation of the NF-кB activation pathway. J Inorg Biochem 2014; 130: 38-51.

25 Jia Z, Nallasamy P, Liu D, Shah H, Li JZ, Chitrakar R et al. Luteolin protects against vascular inflammation in mice and TNF-alpha-induced monocyte adhesion to endothelial cells via suppressing $\mid \mathrm{KB} \alpha / \mathrm{NF}-\kappa \mathrm{B}$ signaling pathway. J Nutr Biochem 2015; 26: 293-302.

26 Shi J, Sun X, Lin Y, Zou X, Li Z, Liao Y et al. Endothelial cell injury and dysfunction induced by silver nanoparticles through oxidative stress via IKK/NF-kappaB pathways. Biomaterials 2014; 35: 6657-6666.

27 Hamuro M, Polan J, Natarajan M, Mohan S. High glucose induced nuclear factor kB mediated inhibition of endothelial cell migration. Atherosclerosis 2002; 162: 277-287.

28 Mohan S, Hamuro M, Koyoma K, Sorescu GP, Jo H, Natarajan M. High glucose induced NF-kB DNA-binding activity in HAEC is maintained under low shear stress but inhibited under high shear stress: role of nitric oxide. Atherosclerosis 2003; 171: 225-234.

29 Lamers D, Schlich R, Horrighs A, Cramer A, Sell H, Eckel J. Differential impact of oleate, palmitate, and adipokines on expression of $\mathrm{NF}-\kappa \mathrm{B}$ target genes in human vascular smooth muscle cells. Mol Cell Endocrinol 2012 362: 194-201.

30 Souza DG, Amaral FA, Fagundes CT, Coelho FM, Arantes RM, Sousa LP et al. The long pentraxin PTX3 is crucial for tissue inflammation after intestinal ischemia and reperfusion in mice. Am J Pathol 2009; 174 1309-1318.

31 Fried LE, Arbiser JL. Honokiol, a multifunctional antiangiogenic and antitumor agent. Antioxid Redox Sign 2009; 11: 1139-1148.

32 Chiang CK, Sheu ML, Hung KY, Wu KD, Liu SH. Honokiol, a small molecular weight natural product, alleviates experimental mesangial proliferative glomerulonephritis. Kidney Int 2006; 70: 682-689.

33 Park J, Lee J, Jung E, Park Y, Kim K, Park B et al. In vitro antibacterial and anti-inflammatory effects of honokiol and magnolol against Propionibacterium sp. Eur J Pharmacol 2004; 496: 189-195.
34 Bai X, Cerimele F, Ushio-Fukai M, Waqas M, Campbell PM, Govindarajan B et al. Honokiol, a small molecular weight natural product, inhibits angiogenesis in vitro and tumor growth in vivo. J Biol Chem 2003; 278: 35501-35507.

35 Ishitsuka K, Hideshima T, Hamasaki M, Raje N, Kumar S, Hideshima H et al. Honokiol overcomes conventional drug resistance in human multiple myeloma by induction of caspase-dependent and-independent apoptosis. Blood 2005; 106: 1794-1800.

36 Zbidah M, Lupescu A, Herrmann T, Yang W, Foller M, Jilani K et al. Effect of honokiol on erythrocytes. Toxicol In Vitro 2013; 27: 1737-1745.

37 Zhu X, Wang Z, Hu C, Li Z, Hu J. Honokiol suppresses TNF- $\alpha$-induced migration and matrix metalloproteinase expression by blocking NF-kB activation via the ERK signaling pathway in rat aortic smooth muscle cells. Acta Histochem 2014; 116: 588-595.

38 Lee B, Kim CH, Moon SK. Honokiol causes the p21WAF1-mediated G(1)phase arrest of the cell cycle through inducing p38 mitogen activated protein kinase in vascular smooth muscle cells. FEBS Lett 2006; 580 5177-5184.

39 Wang JJ, Zhao R, Liang JC, Chen Y. The antidiabetic and hepatoprotective effects of magnolol on diabetic rats induced by high-fat diet and streptozotocin. Yao Xue Xue Bao 2014; 49: 476-481.

40 Zhang $X$, Chen S, Wang Y. Honokiol up-regulates prostacyclin synthease protein expression and inhibits endothelial cell apoptosis. Eur J Pharmacol 2007; 554: 1-7.

41 Livak KJ, Schmittgen TD. Analysis of relative gene expression data using real-time quantitative PCR and the $2-\Delta \Delta$ CT method. Methods $2001 ; 25$ 402-408.

42 Chen LF, Greene WC. Shaping the nuclear action of NF-kappaB. Nat Rev Mol Cell Biol 2004; 5: 392-401.

43 Naugler WE, Karin M. NF-kappaB and cancer-identifying targets and mechanisms. Curr Opin Gene Dev 2008; 18: 19-26.

44 Sheu ML, Chiang CK, Tsai KS, Ho FM, Weng TI, Wu HY et al. Inhibition of NADPH oxidase-related oxidative stress-triggered signaling by honokiol suppresses high glucose-induced human endothelial cell apoptosis. Free Radic Biol Med 2008; 44: 2043-2050.

45 Zhang $\mathrm{P}$, Liu X, Zhu Y, Chen S, Zhou D, Wang Y. Honokiol inhibits the inflammatory reaction during cerebral ischemia reperfusion by suppressing NF-kB activation and cytokine production of glial cells. Neurosci Lett 2013; 534: 123-127.

46 Ahn KS, Sethi G, Shishodia S, Sung B, Arbiser JL, Aggarwal BB. Honokiol potentiates apoptosis, suppresses osteoclastogenesis, and inhibits invasion through modulation of nuclear factor-kappaB activation pathway. Mol Cancer Res 2006; 4: 621-633.

47 Ou HC, Chou FP, Lin TM, Yang CH, Sheu WH. Protective effects of honokiol against oxidized LDL-induced cytotoxicity and adhesion molecule expression in endothelial cells. Chem Biol Interact 2006; 161: 1-13.

48 Ugusman A, Zakaria Z, Chua KH, Nordin NA, Abdullah Mahdy Z. Role of rutin on nitric oxide synthesis in human umbilical vein endothelial cells. $\mathrm{Sci}$ World J 2014; 2014: 169370.

(c) (1) (2) This work is licensed under a Creative Commons Attribution-NonCommercial-ShareAlike 4.0 International License. The images or other third party material in this article are included in the article's Creative Commons license, unless indicated otherwise in the credit line; if the material is not included under the Creative Commons license, users will need to obtain permission from the license holder to reproduce the material. To view a copy of this license, visit http://creativecommons.org/licenses/by-nc-sa/4.0/ 